\title{
EUROPEAN SPINE JOURNAL
}

\section{Expert's comment concerning Grand Rounds case entitled "Malignant triton tumor: grand round presentation of a rare aggressive case thoracolumbar spine tumour."}

\author{
David Choi FRCS PhD
}

The authors report a case of malignant triton tumor of the spine, presenting at the thoracolumbar of a 70 year old gentleman, with back pain and left leg pain of two years duration. Unfortunately, 3 months after complete marginal resection, the patient developed local recurrence and seeding to the cauda equina, and died 10 months after diagnosis.

Malignant triton tumor is a rare variant of malignant peripheral nerve sheath tumor that has rhabdomyoblastic differentiation [1]. The tumor originates from neural crest cells, and is a high grade sarcomatous lesion. Median age at presentation is 29 years, with male to female ratio 1.5:1. Overall survival at 18 months is around $45 \%$, but adverse factors include incomplete resection, spinal involvement, size greater than $2 \mathrm{~cm}$, or association with neurofibromatosis. Accepted treatment is complete excision and post-operative radiotherapy, and sometimes chemotherapy. However, as in this case, resection of extensive spinal tumors is rarely complete.

Although the authors did not perform a pre-operative CT-guided needle biopsy, the article raises the point that if a spine tumor looks unusual, it may be better to perform a biopsy or further staging investigations prior to removal, unless the patient presents as an emergency with rapid neurological progression. All spine tumors should be discussed in a multidisciplinary setting, with input from oncologists, spine surgeons, radiologists and pathologists. Particularly for unusual cases, multiprofessional discussion should increase the likelihood of choosing the most appropriate treatment, ensures co-ordination of treatment, and the delivery of consistent information to the patient [2]. Needle biopsies are generally safe, and with newer biopsy needles (thinner gauge, sheathed needles) they are less likely to be associated with tumor seeding along the biopsy tract [3]. The information obtained from the biopsy may alter the surgical plan or inform the consent process. Some tumors may respond better to primary chemotherapy or radiotherapy before surgery, or instead of surgery. Other tumors may have such a poor prognosis or high recurrence rate that surgery is not advisable, or is unlikely to change the natural history of the disease. Staging investigations are also important (for example, CT chest, CT Positron emission tomography, or whole body MRI) and will influence the surgical decision making and proposed extent of surgery. For example, it would be futile to perform a complex en bloc resection of spinal tumor in the presence of suspected metastatic disease.

In the present case, more extensive surgery may not have changed the outcome, and marginal excision of the tumor was the likely treatment of choice. For intradural tumors, it is not possible to perform en bloc resection without sacrificing neurological function, and clear oncological clearance is seldom possible. The authors should be congratulated for highlighting the possibility of a rare aggressive tumor masquerading as a common benign tumor, and the possible management options should be considered at an early stage. If a pre-operative diagnosis of malignant tumor had been made by biopsy, consideration might be given to more extensive resection including T12 nerve root and adjacent vertebral bone and paraspinal soft tissues, or alternatively the option of a more palliative management plan, in some circumstances, would be appropriate. All potential options, as well as the recommended management plan, should be discussed with the patient during the consent process [4].

\section{References}

1. Prieto R, Pascual JM, Garcia-Carbezas MA, Lopez-Barea F, Barrios L, Gonzalez-Llanos F (2012) Low-grade malignant triton tumor in the lumbar spine: a rare variant of malignant peripheral nerve sheath tumor with rhabdomyoblastic differentiation. Neuropathology 32(2):180-189

2. Fleissig A, Jenkins V, Catt S, Fallowfield L (2006) Multidisciplinary teams in cancer care: are they effective in the UK? Lancet Oncology 7(11):935-943 
3. Tyagi R, Dey P (2014) Needle tract seeding: an avoidable complication. Diagnostic Cytopathology 42(7): 636-640

4. Chan SW, Tulloch E, ,Cooper ES, Smith A, Wojcik W, Norman JE (2017) Montgomery and informed consent: where are we now? British Medical Journal May 12;357:j2224. doi: 10.1136/bmj.j2224 